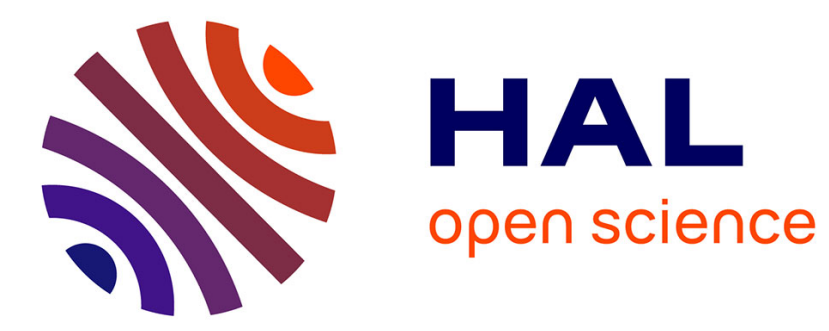

\title{
Modélisation géométrique 3D des granites stéphaniens du massif du Pelvoux (Alpes, France).
}

Pierre Strzerzynski, Stéphane Guillot, Gabriel Courrioux, Patrick Ledru

\section{To cite this version:}

Pierre Strzerzynski, Stéphane Guillot, Gabriel Courrioux, Patrick Ledru. Modélisation géométrique 3D des granites stéphaniens du massif du Pelvoux (Alpes, France).. Comptes Rendus Géoscience, 2005, 337, pp.1284-1292. 10.1016/j.crte.2005.06.008 . hal-00103175

\section{HAL Id: hal-00103175 https://hal.science/hal-00103175}

Submitted on 5 Oct 2006

HAL is a multi-disciplinary open access archive for the deposit and dissemination of scientific research documents, whether they are published or not. The documents may come from teaching and research institutions in France or abroad, or from public or private research centers.
L'archive ouverte pluridisciplinaire HAL, est destinée au dépôt et à la diffusion de documents scientifiques de niveau recherche, publiés ou non, émanant des établissements d'enseignement et de recherche français ou étrangers, des laboratoires publics ou privés. 
Rubrique : Tectonique

Modélisation géométrique 3D des granites Stéphaniens du massif du Pelvoux (Alpes, France).

3D geometrical modelling of Stephanian granite from the Pelvoux massif (French Alps).

Pierre Strzerzynski ${ }^{(1)}$, Stéphane Guillot ${ }^{(1)}$, Gabriel Courrioux ${ }^{(2)}$, Patrick Ledru ${ }^{(1-2)}$

(1) : Laboratoire de Sciences de la Terre, CNRS UMR 5570, UCB-ENS Lyon, Bât. Géode, 2, rue Dubois, 69622 Villeurbanne cedex, FRANCE

(2) : CDG-MA, BRGM, avenue C. Guillemin, 45060 Orléans, France

Mots clés : Alpes, granite, modélisation 3D, Carbonifère.

Key words : Alps, Granite, 3D modelling, Carbonifere.

Correspondance : pierre.strzerzynski@univ-lyon1.fr 
Résumé

La modélisation 3D de la géométrie des granites Stéphaniens du Massif du Pelvoux a permis de mettre en évidence le contexte cisaillant associé à leur mise en place. Dans les Massifs Cristallins Externes Français, ces cisaillements se répartissent selon deux directions, N50 et N135, respectivement dextre et sénestre. Ce système décrochant Carbonifère s'intègre dans un contexte d'extension N-S connu dans l'ensemble de la chaîne Varisque.

Keyword: Western Alps, Granites, 3D modelling, tectonics, Carboniferous.

Abstract

3D modelling of Stephanian granites of the Pelvoux massif characterizes an emplacement along sinistral NW-SE and dextral NE-SW trending shear zone respectively in the Pelvoux and in the Aiguilles Rouges-Mont Blanc massifs. This Carboniferous shear system is consistent with a N-S extension direction known in the whole Variscan belt. 


\section{Abridged English Version}

Introduction

Mechanisms of granite emplacement are characteristic of the tectonic contexts that are active during orogens, especially for the Variscan orogeny, fertile in granite. Their knowledge requires a modelling of the geometry of the massifs and a structural analysis of their relation with their host rocks. Within eroded belts, as high-resolution geophysical surveys or deep drilling are rare, 3D modelling remains highly speculative. However, reactivation and exhumation of Paleozoic basement during the Alpine orogeny, provides access to about $2000 \mathrm{~m}$ fall of level within the External Crystalline Massif of the French Western Alps (figure I).

This paper describes the 3D modelling of the Turbat, Etages and Berarde granites located in the Pelvoux Massif. An analysis of their structural relationships with their host rocks is presented and their mode of emplacement is discussed. As a significant rotation of these massifs is excluded since Trias [1], the sinistral strike slip component determined along NW-SE structures controlling granite emplacement is integrated in the Variscan scheme.

1. The granites of the Pelvoux massif

\subsection{Geological context}

The Pelvoux Massif is located in the western Alps (France). It forms with the Mont Blanc, Aiguilles Rouges, Belledonne and Argentera Massif the External Crystalline Massifs. They mainly consist of micaschists, para and orthogneiss intruded by Carboniferous granites and exhumed $15 \mathrm{Ma}$ ago along major structural discontinuities (Figure I) [2-3].

Their structural and metamorphic evolution are related to the Variscan orogeny and correlated to the French Massif Central, Vosges and Bohemian massif [18]. The tectonic evolution of these Palaeozoic domains begins with the formation of an ocean during Cambrian and Ordovician. A subduction is then recorded during the lower Devonian [17] followed by a collision phase marked by nappe stacking [12,9] and emplacement of K-Mg rich 
granites [6]. During Upper Carboniferous, extensional structures [12, 9], high geothermal gradient [10] and emplacement of Fe-Mg granite [6] traduce post collision evolution and mark the progressive return to normal thickness of the crust.

\subsection{The Pelvoux External Crystalline Massif}

The Pelvoux Massif has a sub-circular shape consisting of two different litho-structural units (figure I): the eastern and central parts are mainly composed of migmatite and granites while the western part presents low to medium-grade metasediments. Two migmatitic stages are recognized [3] developed prior to the emplacement of granites. The studied granites are located at the centre of the Pelvoux Massif, intuding migmatitic orthogneiss (metagranite and amphibolite) and paraneiss.

\subsection{The Turbat, Etages and Berarde granites}

On the basis of chemical analogy, these three granites belong to the Fe-Mg granite suite, emplaced during the Stephanian [6]. The Turbat granite is dated at $302 \pm 2 \mathrm{Ma}$ [U-Pb, zircon, 11]. The granites have a NNW-SSE elongated shape truncated on their northern margin by a tectonic limit: the Meije-Muzelle trust that affects Mesozoic sedimentary sequences [4] (Figure I). Southward, the roof of the intrusion is exposed and primary intrusive contacts between the granites and their host rocks are preserved.

The feldspar, biotite and enclave preferred orientation defines a vertical sub-solidus magmatic foliation oriented N160 (figure II) fairly constant within the three massifs. A horizontal mineral lineation is visible on this plane. The foliation planes are locally grading into zones of intense deformation marked by vertical C-S shear zones oriented N135 (Figure II) indicating a sinistral movement.

\section{3D modelling}

\subsection{Principle}

The 3D geometrical modelling software "Editeur Geologique" has been developed in the framework of the BRGM research programs. That software, conceived for being used by geologists, allows the building the 3D shape of geological object on the basis of datasets introduced into interface/buffer as map or cross section (figure III) and visualized in 2 (figure IV) and 3D viewers (Figure V). The data used to constrain the model are:

- the lithologies and lithological contacts defined by few points, 
- the topological relationships between the different lithological units, the geometrical relationship like superposition, intrusion or cross cutting relation can be integrated though the definition of the lithological pile,

- The orientation of planar structures like foliation, schistosity or stratification or linear structures as fold axes.

(Figure III). The 3D geometrical modelling consist of an interpolation of a scalar field in the space where a lithological contact corresponds to an isovalue of this field and the orientation of the structure corresponds to the gradient of this field [13].

\subsection{The $3 \mathrm{D}$ modelling of the Pelvoux granites}

The 3D shape of the Turbat, Etages and Berarde granite has been built using three kinds of surfaces, through three successive stages of modelling (Figure III, IV and V):

- The N160-vertical foliation, developed during granite emplacement, is considered to be representative and sub-parallel to the initial intrusive contacts (Figure IV). Taking into account the vertical distribution of the measured structures, this first model provides a well-constrained model of the eastern and western margin of the granites.

- The second stage of modelling takes into account the granite-gneiss contact at the roof of the granite. The plunge of this contact remains low, respectively northward and southward on the northern and southern margin of the granite. The foliation within the granite presents a high obliquity with the contact at the roof of the granite and the modelling is performed through direct construction on the Digital Elevation Model, taking into account structural data measured on the contact and extrapolation following its regional extent.

- $\quad$ In the late stage, the Meije-Muzelle thrust zone is built (Figure V). This alpine tectonic zone represents the northward boundary of the granites cross cutting the foliation within the granites. The modelling is performed by defining the trace and structural orientation of the fault.

\section{3. discussion}

\subsection{The alpine deformation of the Pelvoux Massif}


The Meije-Muzelle thrust zone constitutes the main trace of alpine tectonics, marking the northern limit of the studied granites (figure V). Mesozoic sedimentary sequences pinched within that accident attest of the alpine age of this accident [4]. Local reactivations of primary magmatic contacts between the granites and their host rocks occur first during the Liassic extension event [16] and are inverted during the alpine orogeny (figure IV).

\subsection{The mechanisms of granite emplacement}

The Pelvoux granites have eastern and western vertical boundaries and display a N160 elongated shape corresponding to the development of a foliation synchronous of granite crystallisation. The association of this foliation to NW-SE sinistral shear zones favours an emplacement of the granites during a transcurrent tectonic context. That shearing context can also be observed in the host rocks or in the Peyre-Arguet unit located in the SW of the Pelvoux massif (figure I) [10]. At the scale of the Alps, this NW-SE direction corresponds to the southern trend of the Alpine Arc, from the Pelvoux to the Argentera.

North of this area, emplacement of the Stephanian Vallorcine and Mont Blanc granites has been dated at 306 and $303 \mathrm{Ma}$ [5]. That magmatic event is associated with a dextral NE-SW shear movement within a generalised extensional tectonic context [5]. The opposite sense of shear and the chemical analogy and synchronism of the Mont Blanc and Pelvoux granites suggest that these two strike slip directions could be conjugated, during a bulk regional EW shortening and N-S extension (figure VI). This strain field is also compatible with the E-W trending stephanian lamprophyre dyke, post dating the emplacement and cooling of the granites [14]. This extension direction is attributed to the Stephanian post-collision stage of extension identified at the scale of the Variscan belt [7].

\subsection{The integration within the European Palaeozoic belt}

The External Cristalline Massifs and the French Massif Central have similar Palaeozoic evolution [7]. However this study highlights difference during the late stage of the Palaeozoic evolution. The French Massif Central granites are characterised by dome shape and laccoliths geometry emplaced during N-S extension direction in a context of crustal thinning $[8,15]$ whereas granites of the External Crystalline Massifs emplaced along dextral and sinistral shear zone. Although the direction of extension is similar, shortening is vertical in the French Massif Central and E-W into the External Crystalline Massif. As the External Crystalline Massifs are located on 
the Eastern margin of the European Palaeozoic belt, they may have been perturbed by the rotation translation of Africa relative to the Europe [2], associated with the NW oblique subduction of the Paleo-Tethys [19].

conclusion

The 3D modelling of the granites of the Pelvoux Massif reveals that the overall shape of the intrusion, determined from internal structures and relationships with their host rocks, is compatible with an emplacement along a sinistral NW-SE trending strike-slip. At the scale of the External Crystalline Massif, this context corresponds to a bulk regional EW compression and NS extension marked by N50 dextral and N140 sinistral conjugated shear zones, respectively in the Mont Blanc and Pelvoux massifs. 
Introduction

Au sein des orogenèses, le mode de mise en place des granites nous renseigne sur le contexte tectonique global. Ceci est particulièrement vrai dans le cas de la chaîne Paléozoïque Européenne, fertile en granites. La connaissance des massifs granitiques nécessite l'établissement d'un modèle géométrique et l'analyse des relations structurales avec leur encaissant. Dans les chaînes anciennes et érodées et en l'absence de données géophysiques à haute résolution, la géométrie des corps granitiques est souvent difficile à définir en trois dimensions. La réactivation et la mise en relief du socle Paléozoïque des Massifs Cristallins Externes des Alpes occidentales (figure I) offrent des conditions exceptionnelles d'analyse en trois dimensions. Des données structurales peuvent ainsi être acquises sur plus de $2000 \mathrm{~m}$ de dénivelé donnant accès à la géométrie en profondeur du massif et des grandes discontinuités géologiques.

Le mode de mise en place des massifs granitiques de Turbat Lauranoure, des Etages et de la Bérarde (Massif du Pelvoux et des Ecrins) est étudié dans le cadre d'une modélisation géométrique 3D d'un domaine de $18 \mathrm{~km} \mathrm{x}$ $18 \mathrm{~km}$ x $8 \mathrm{~km}$ en $\mathrm{X}$, Y et Z. Les données acquises et le modèle résultant fournissent des contraintes géométriques fiables sur la structure de la croûte supérieure. Les granites s'enracinent verticalement sur plusieurs kilomètres et définissent un contexte de mise en place dans un environnement décrochant senestre selon une direction N135E. En l'absence de rotation significative des Massifs Cristallins Externes depuis le Trias [1], ces résultats sont intégrés dans le schéma d'évolution de la chaîne Paléozoïque Européenne.

1. Le massif du Pelvoux

\subsection{Contexte géologique régional}

Le Massif du Pelvoux occupe une position centrale dans les Massifs Cristallins Externes des Alpes Occidentales, il est prolongé au NE par le Massif de Belledonne et au SE par le Massif de l'Argentera (figure I). Ces terrains représentent des fragments de la chaîne Paléozoïque Européenne [18], réactivés lors des compressions alpines le long de grandes discontinuités structurales puis exhumés il y a environ 15 Ma [20].

L’évolution Paléozoïque des Massifs Cristallins Externes débute par une phase d'océanisation CambroOrdovicienne qui isole un bloc Armorica du Gondwana. Au Dévonien inférieur et moyen, cet océan entre en subduction [17]. Le Dévonien supérieur correspond à une phase de collision responsable de l'empilement de nappes crustales [12,9] et à la mise en place de granites potassiques [6]. L'évolution tardi-orogénique des 
Massifs Cristallins Externes se marque au Carbonifère supérieur par des structures d'extension dans le massif de Belledonne [12, 9], un gradient géothermique anormalement élevé dans le massif du Pelvoux [10] et un magmatisme important auquel se rattache les massifs granitiques étudiés [6].

\subsection{Les granites du Turbat-Lauranoure, des Etages et de la Bérarde}

Les granites Turbat-Lauranoure, des Etages et de la Bérarde forment la partie centrale du Massif du Pelvoux. Ils sont intrusifs dans des gneiss migmatitiques et des amphibolites (figure I) au sein desquels deux épisodes de métamorphisme haute température anté-granite sont reconnus [3]. Ces trois granites sont chimiquement rattachés à la même suite magmatique Fe-Mg mise en place au Stéphanien [6]. Ainsi, la mise en place du granite du Turbat est datée à 302 +/-2 Ma [11] (U-Pb sur zircon). En carte (Figure I), ces granites montrent un allongement selon une direction N160E. Ils sont limités au nord par le chevauchement Meije-Muzelle qui implique des sédiments Mésozoïques [4]. Au sud, les granites disparaissent sous les gneiss migmatitiques. L'analyse structurale révèle la présence d'une foliation pénétrative orientée N160E, sub-verticale. Cette foliation est marquée par l'allongement préférentiel des feldspaths, des biotites et des enclaves basiques, elle affecte de façon homogène les trois massifs granitiques (figure II et III). Une linéation d'étirement sub-horizontale est localement observée dans le plan de foliation. Des bandes de cisaillement ductile d'orientation N135E et verticales sont visibles (figure II). Les structures C-S apparaissent en continuité structurale avec la foliation dominante des granites et traduisent un mouvement décrochant senestre. Enfin, des filons de basalte, orientés globalement E-W et mis en place au Trias, sont exempts de déformations et recoupent les structures plano-linéaires des granites. Ces caractères indiquent que la structuration des massifs granitiques a été acquise lors de leur cristallisation et peut donc être considérée comme un marqueur du mode de mise en place.

\section{2. modélisation 3D}

\subsection{Principe de cartographie numérique 3D}

L'Editeur Géologique 3D a été développé au BRGM dans une perspective de cartographie 3D prenant en compte la géométrie des objets géologiques en profondeur. La construction des modèles est faite par le géologue à partir de cartes et de coupes géologiques visualisées en 2D (figure III), le modèle résultant étant visualisé à chaque étape de la construction sur ces mêmes supports 2D (figures IV) et en 3D (figure V).

Les données utilisées sont : 
- la localisation géographique des points de passage des interfaces, contacts lithologiques normaux ou faillés ;

- les mesures d'orientation de l'anisotropie principale, stratification ou foliation, d'une densité et répartition significative à l'échelle du domaine modélisé (figure III).

La construction du modèle 3D se fait par interpolation d'un champ scalaire dans l'espace pour lequel les points de passage d'une même interface lithologique ont la même valeur et où les données d'orientation représentent le gradient [13]. La position des données d'orientation est indépendante de la localisation des interfaces. Les implications géométriques de la nature de certains contacts, telles que la superposition, l'érosion et l'intersection ou encore inclusion ou intrusion, sont prises en compte dans la modélisation (figure III).

\subsection{Reconstruction de la géométrie des granites du Pelvoux}

La procédure mise en œuvre pour la construction du modèle géométrique 3D des granites du TurbatLauranoure, des Etages et de la Bérarde est la suivante (figure II, III, IV et V).

Un premier modèle résulte de l'interpolation d'un champ scalaire défini par les mesures de foliation des massifs granitiques. Ce modèle permet de définir, en profondeur, la géométrie des limites est et ouest des granites. Leur orientation est N160E et leur pendage est vertical sur au minimum deux kilomètres de profondeur (figure 5). Ces surfaces correspondent à des contacts granite-gneiss à l'exception du contact entre les granites des Etages et de la Bérarde. Ce sont des contacts magmatiques développés lors de la mise en place des granites. Ce premier modèle permet de mettre en évidence la géométrie allongée des granites selon une direction N160E cependant les limites nord et sud ne sont pas prises en compte.

Un second modèle est calculé afin d'intégrer les contacts granites-gneiss qui couronnent certains sommets (tête du Rouget, Aiguille du Plat de la Selle, tête du Salude) et la limite sud des granites. La morphologie exacte de ces contacts supérieurs est difficile à contraindre. En effet le toit du granite est souligné sur le terrain par un réseau diffus de filons granitiques intrusifs dans les gneiss. De ce fait, le toit du granite a été construit en fournissant des données moyennes d'orientation de ce contact à l'échelle de la carte et du modèle. Ce contact présente un faible pendage vers le nord au nord du massif et vers le sud au sud.

Un troisième modèle a été réalisé afin de rendre contre de la géométrie du chevauchement de Meije-Muzelle (figure 6). Sur le terrain, ce contact est orienté N50E-50 ${ }^{\circ} \mathrm{SE}$. Il constitue la limite nord des granites du TurbatLauranoure et des Etages et il se prolonge dans les gneiss entre ces deux granites. Ce contact est sécant sur la foliation du granite. 
3. discussion

\section{1. signification des contacts tardifs}

La limite nord des granites correspond à des accidents chevauchants orientés NE-SW dont le mouvement est alpin [4]. Observés sur plus de $1500 \mathrm{~m}$ de dénivelé, ces accidents montrent un pendage constant, d'environ $50^{\circ}$ vers le sud-est. Sur les modèles 3D (figure V), ces accidents ont une géométrie plane et ils sont systématiquement recoupés par les limites est et ouest des granites. Ces observations suggèrent que les anciens contacts magmatiques ont été réactivés, tout d'abord lors de l'extension Liasique, donnant naissance à des failles normales pentées vers le SE, puis inversés en régime compressif lors du cycle orogénique alpin [16].

3.2. Contexte de mise en place des granites du Pelvoux

Les bordures rectilignes et de pendage raide, donnent aux granites du massif du Pelvoux une géométrie allongée selon une direction $\mathrm{N} 160 \mathrm{E}$ en bande verticale. Cette géométrie est en accord avec les données structurales qui indiquent un contexte de mise en place dans des décrochements sénestres de direction N135E. A l'échelle des Alpes, cette direction de cisaillement correspond à la direction de l'axe Pelvoux-Argentera.

Plus au nord, dans les Massifs Cristallins Externes du Mont Blanc et des Aiguilles Rouges, la mise en place des granites du Mont Blanc et de Vallorcine (figure VI) a été datée au Carbonifère Supérieur respectivement à 303 et $306 \mathrm{Ma}$ [5]. Ces granites se mettent en place à la faveur de décrochements dextres de direction N50E dans un contexte d'extension généralisé [5]. A l'échelle des Massifs Cristallins Externes, ces deux directions de décrochement (NW-SE et NE-SW) sont contemporaines. Leur sens opposé et la mise en place de granites de chimie analogue [6] font de ces grands décrochements des structures conjuguées d'échelle crustale (plus de 300 $\mathrm{km}$ de long) compatibles avec une direction d'extension N-S et une direction de raccourcissement E-W. Ces même directions d'extension et de raccourcissement ont été déterminées grâce à l'orientation E-W des filons de lamprophyres mis en place dans les massif du Pelvoux [14] et de l'Argentera au Carbonifère Supérieur. Enfin, cette direction d'extension est proche de celle décrite dans le massif de Belledonne pour la même période [9].

3.3. intégration au sein de la chaîne Paléozoïque 
L’évolution Paléozoïque des Massifs Cristallins Externes est en de nombreux points comparable à celle connue dans le Massif Central Français [7] : les phases d'océanisation et de subduction [17] puis de collision suivis d'extension $[12,9]$ ont été documentés dans les massifs de Belledonne et du Pelvoux.

Cependant, cette étude met en évidence des différences dans les dernières étapes du cycle Paléozoïque, au cours du Stéphanien. En effet, les granites du Massif Central Français tel que le massif du Velay sont caractérisés par des géométries de dômes et de laccolites s'inscrivant dans un contexte d'amincissement crustal avec une direction d'extension principale orientée nord-sud [8, 15]. Dans les Massifs Cristallins, les décrochements conjugués dextre et senestre indiquent aussi une direction d'extension nord sud, mais la direction de raccourcissement n'est plus verticale, mais horizontale et de direction est-ouest. Cette différence dans l'orientation de la composante de raccourcissement traduit la position plus marginale des Massifs Cristallins Externes par rapport au cœur de la chaîne. Ils sont ainsi impliqués dans la rotation et la translation dextre de l'Afrique par rapport à l'Europe [2] qui a été récemment interprétés comme résultant de la subduction oblique de la Paléo-Téthys vers le nord-Ouest [19]. Ce contexte de subduction oblique pourrait être à l'origine de la mise en place de ces granites au sein d'une croûte préalablement épaissie dans un contexte tectonique décrochant [5].

4. conclusion

Malgré une réactivation au cours du cycle Alpin, l'analyse structurale et la cartographie 3D des granites du Massif du Pelvoux ont permis de mettre en évidence le contexte décrochant responsable de leur mise en place au cours du Stéphanien. Dans les Massifs Cristallins Externes, ces décrochements se répartissent selon deux familles : ils sont orientés N50E et de composante dextre dans le massif du Mont Blanc et N135E avec une composante sénestre dans le massif du Pelvoux. Ces directions de cisaillement sont compatibles avec un raccourcissement est-ouest et une extension nord-sud. La prédominance des systèmes décrochants dans les Massifs Cristallins Externes au cours du Stéphanien, est expliquée par leur situation en bordure de la chaîne Paléozoïque Européenne, à proximité de la zone de subduction oblique de la Paléo-Téthys.

\section{Remerciements}

Les auteurs remercient Arnaud Pêcher pour ses remarques constructives. Ce travail a été financé par le projet INSU--BRGM "GéoFrance-3D Alpes" 


\section{Références}

[1] C. Aubourg, C. Chabert-Pelline. Neogene remagnetization of normal polarity in the Late Jurassic black shales from the southern subalpine chains (French Alps); evidence for late anticlockwise rotations. Tectonophysics, 308 (1999) 473-486.

[2] J. P. Bard, Demembrement ante-mesozoique de la chaine varisque d'Europe occidentale et d'Afrique du Nord; role essentiel des grands decrochements transpressifs dextres accompagnant la rotation-translation horaire de l'Afrique durant le Stephanien, C.R. Acad. Sciences, Serie II. 324 (1997) 693-704.[

[3] J. C. Barféty, A. Pecher, notice de la carte géologique au $50000^{\mathrm{eme}}$ de Saint Christophe en Oisans, édition du BRGM.

[4] F. Bartoli, A. Pêcher, P. Vialon, Le chevauchement Meije-muzelle et la répartition des domaines structuraux du massif de l'Oisans (partie du Haut-Dauphiné cristallin). Géologie Alpine, 50 (1974) 17-26.

[5] F. Bussy, J. Hernandez, J. von Raumer, Bimodal magmatism as a consequence of the post-collisional readjustment of the thickened Variscan continental lithosphere (Aiguilles Rouges-Mont Blanc massifs, Western Alps). GSA, 350 (2001) 221-233.

[6] F. Debon, M. Lemmet. Evolution of $\mathrm{Mg} / \mathrm{Fe}$ ratios in late Variscan plutonic rocks from the external crystalline massifs of the Alps (France, Italy, Switzerland). Journal of Petrology 40 (1999) 1151-1185.

[7] S. Di paola, S. Guillot, P. Ledru, R.P. Menot, A.P. Montjoie, M. I. Spalla, G. Gosso, Evidence for a main paleozoic sutur zone in the Belledonne-Grandes-Rousses Oisans Massifs (Western Alps) in the light of Geographical Information System mapping, tectonophysics “in press”.

[8] M. Faure, Late orogenic Carboniferous extensions in the Variscan French Massif Central, Tectonics 14 (1995) 132-153.

[9] A. Fernandez, S. Guillot, R.-P. Menot, P. Ledru. Late Paleozoic polyphased tectonics in the SW Belledonne massif (external crystalline massifs, French Alps). Geodinamica Acta 15 (2002) 127-139.

[10] V. Grandjean, S. Guillot, A. Pecher. Un nouveau témoin de l'évolution métamorphique BP-HT postorogénique hercynienne; l'unité de Peyre-Arguet (Haut-Dauphine). C.R. Acad. Sciences, Série II 322 (1996) 189-195.

[11] C. Guerrot, F. Debon. U-Pb zircon dating of two contrasting late Variscan plutonic suites from the Pelvoux Massif (French Western Alps).” Schweiz. Min. Petr. Mitt. 80 (2000) 249-256.

[12] S. Guillot, and R.-P. Menot. "Nappe stacking and first evidence of late Variscan extension in the Belledonne Massif (External Crystalline Massifs, French Alps).” Geodinamica Acta 12 (1999) 97-111. 
[13] C. Lajaunie, G. Courrioux, L. Manuel, Foliation fields and 3D cartography in geology; principles of a method based on potential interpolation, Mathematical Geology 29 (1997) 571-584.

[14] J. C. Laurent, Les épisodes du magmatisme filonien basique du massif des Ecrins-Pelvoux entre le Carbonifère et le Lias, thèse de troisième cycle, université de Grenoble (1992).

[15] P. Ledru, , G. Courrioux, C. Dallain, J. M. Lardeaux, J. M. Montel, O. Vanderhaeghe and G. Vitel. The Velay Dome (French Massif Central); melt generation and granite emplacement during orogenic evolution, Tectonophysics, 342 (2001) 207-237.

[16] M.T. Lemoine, A. Bas, H. Arnaud-Vanneau, Arnaud, T. Dumont, M. Gidon, M. Bourbon, P.-C. de Graciansky, J.-L. Rudkiewicz, J. Megard-Galli and P. Tricart, The continental margin of the Mesozoic Tethys in the Western Alps, Marine and Petroleum Geology 3 (1986) 179-199.

[17] J.L. Paquette, R.-P. Menot, J.-J. Peucat. "REE, Sm-Nd and U-Pb zircon study of eclogites from the Alpine external massifs (Western Alps); evidence for crustal contamination.” EPSL 96(1989) 181-198.

[18] J. F. von Raumer, The Palaeozoic evolution in the Alps; from Gondwana to Pangea, Geologische Rundschau, 87 (1998) 407-435.

[19] G. M. Stampfli, G. D. Borel. A plate tectonic model for the Paleozoic and Mesozoic constrained by dynamic plate boundaries and restored synthetic oceanic isochrones, E.P.S.L. 196 (2002) 17-33.

[20] Tricart P., Schwartz S., Sue C., Poupeau G. and Lardeaux J.M. La dénudation tectonique de la zone ultradauphinoise et l'inversion du Front Briançonnais au sud-est du Pelvoux (Alpes occidentales): une dynamique miocène 
Figure I: Schéma structural du massif du Pelvoux [3]. 1: granites Viséens, 2: granites Stéphaniens, 3 : couverture sédimentaire Mésozoique, $4:$ gneiss migmatitique et amphibolites, $5:$ Cortex et $6:$ Alpes Internes. Le cadre correspond à la zone couverte par la carte 3D avec A : le granite du Turbat-Lauranoure, B : le granite des Etages et $\mathrm{C}$ : le granite de la Bérarde. Cercles noirs : reliques de granulite [10]

Figure I : Structural map of the Pelvoux massif [3].

1: Visean granites, 2 : Stephanian granites, 3 : Mesozoic sedimentary cover, $4:$ magmatic and amphibolite gneisses, 5 : external zone and $6:$ internal zone of the Alps. The black square represents the surface covered by 3D map. A : Turbat-Lauranoure, B : Etages and C : La Berarde are the modelized granites. Black circles : granulite relics [10]

Figure II : a) Mesures de foliation dans les granites de la Bérarde, des Etages et du Turbat (114 valeurs) et b) mesures des plans de cisaillement (36 valeurs) représentées sur des canevas de Wulf, hémisphère inférieur, contour Kamb.

Figure II: a) Foliation measurements (114 measures) and b) shear plane measurements (36 measures) from Berarde, Turbat and Etages Granites. (Wulf canevas, lower hemisphere, Kamb contouring).

Figure III : Vue en carte des données prises en compte dans la conception du modèle 3D : 1 : points de passages d'une interface, 2 : mesures structurales et $\mathrm{AB}$ trace de la coupe (figure VI). Sont représentés en rouge les bordures est et ouest des granites du Turbat Lauranoure (à l'ouest) et des Etages (à l'est).

Figure III : field and cartographic data set taking into account in the 3D modelling : A: localization of interface, $\mathrm{B}$ : structural measurement and $\mathrm{AB}$ : localization of the cross section. In red: the eastern and western boundarie of the Turbat-Lauranoure granite and Etages granite, in purple: the western la Berarde granite boundary.

Figure IV : vue en carte du modèle 3D calculé.

Figure IV: upper view of the 3D model. 
Figure V : vue vers le nord-ouest de la carte 3D des granites du Turbat, des Etages et de la Bérarde.

Figure $\mathrm{V}: \mathrm{N}-\mathrm{W}$ view of the 3D model.

Figure VI : Schéma structural des massifs cristallins externes français [6] montrant les directions de cisaillement liés à la mise en place des granites au Stéphanien dans le massif du Mont Blanc [5] et dans le massif du Pelvoux.

Figure VI : Structural map of the French External Crystalline massifs [6]. Two shearing directions associated with Stephanian granites emplacement are distinguished: a dextral one located in the Mont Blanc massif [5] and a sinistral one in the Pelvoux massif. 


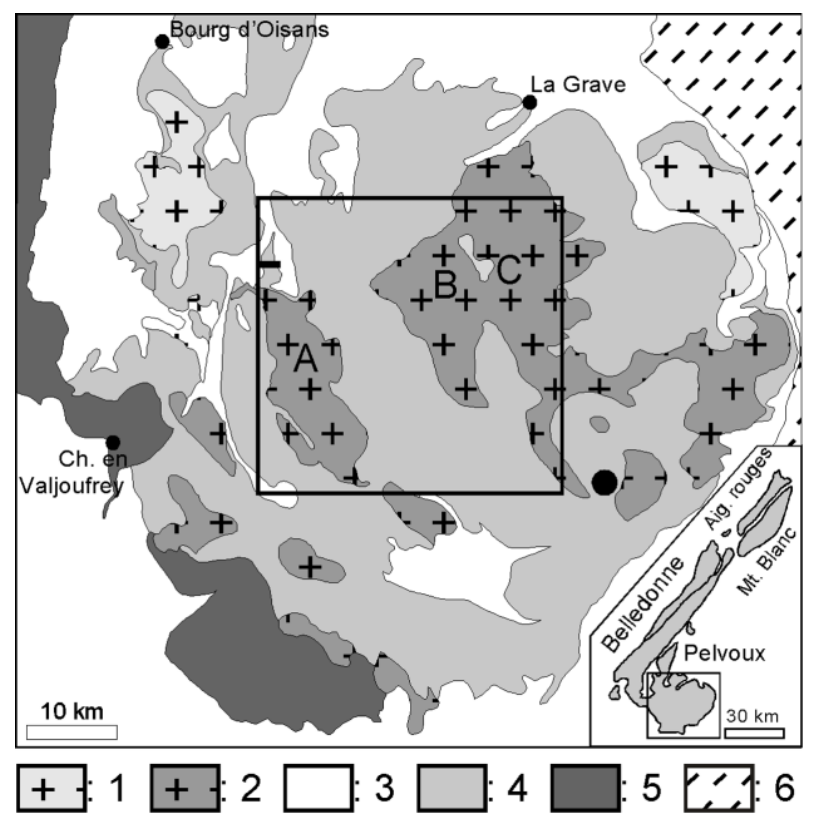

Figure I

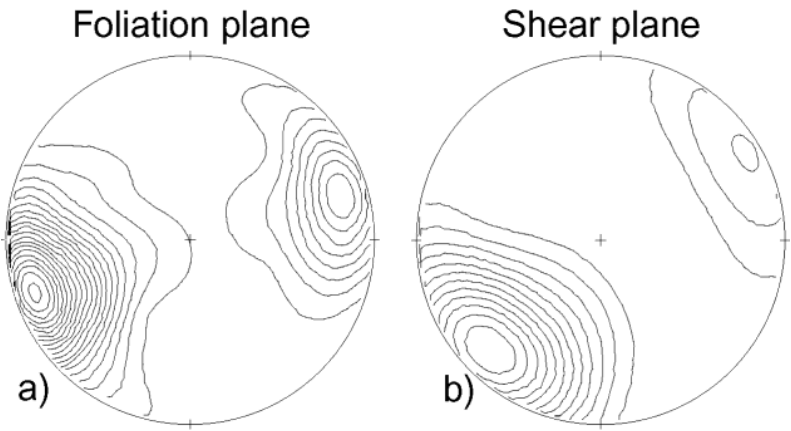

Figure II 


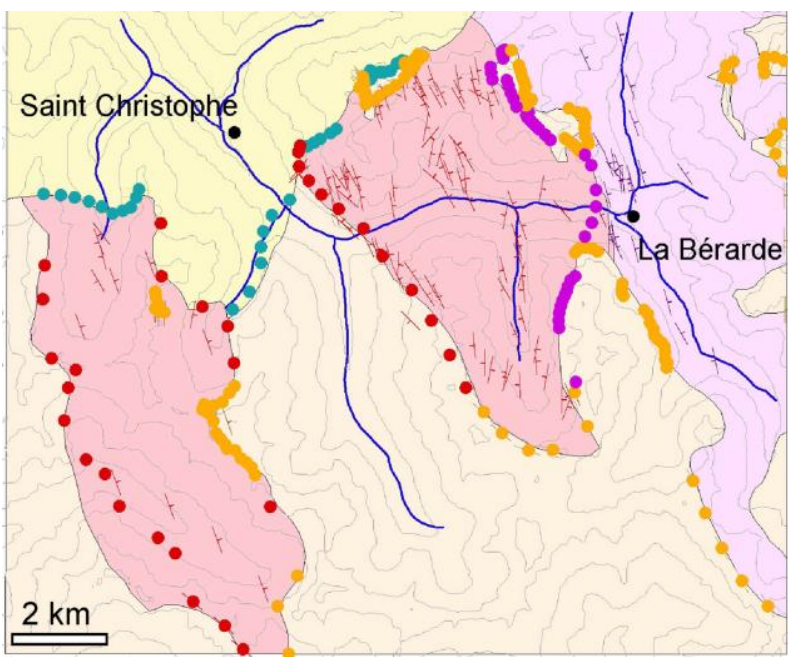

Figure III

Bérarde Granite western boundarie

Eastern and western Granite gneisses boundaries

Roof of the granite

Meije Muzelle Thrust

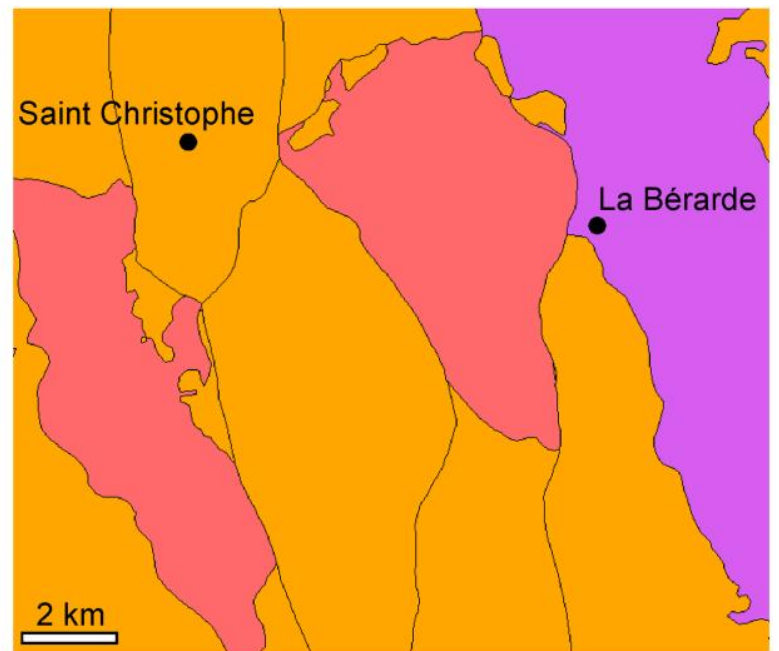

Figure IV gneisses Turbat and Etages Granite Bérarde Granite

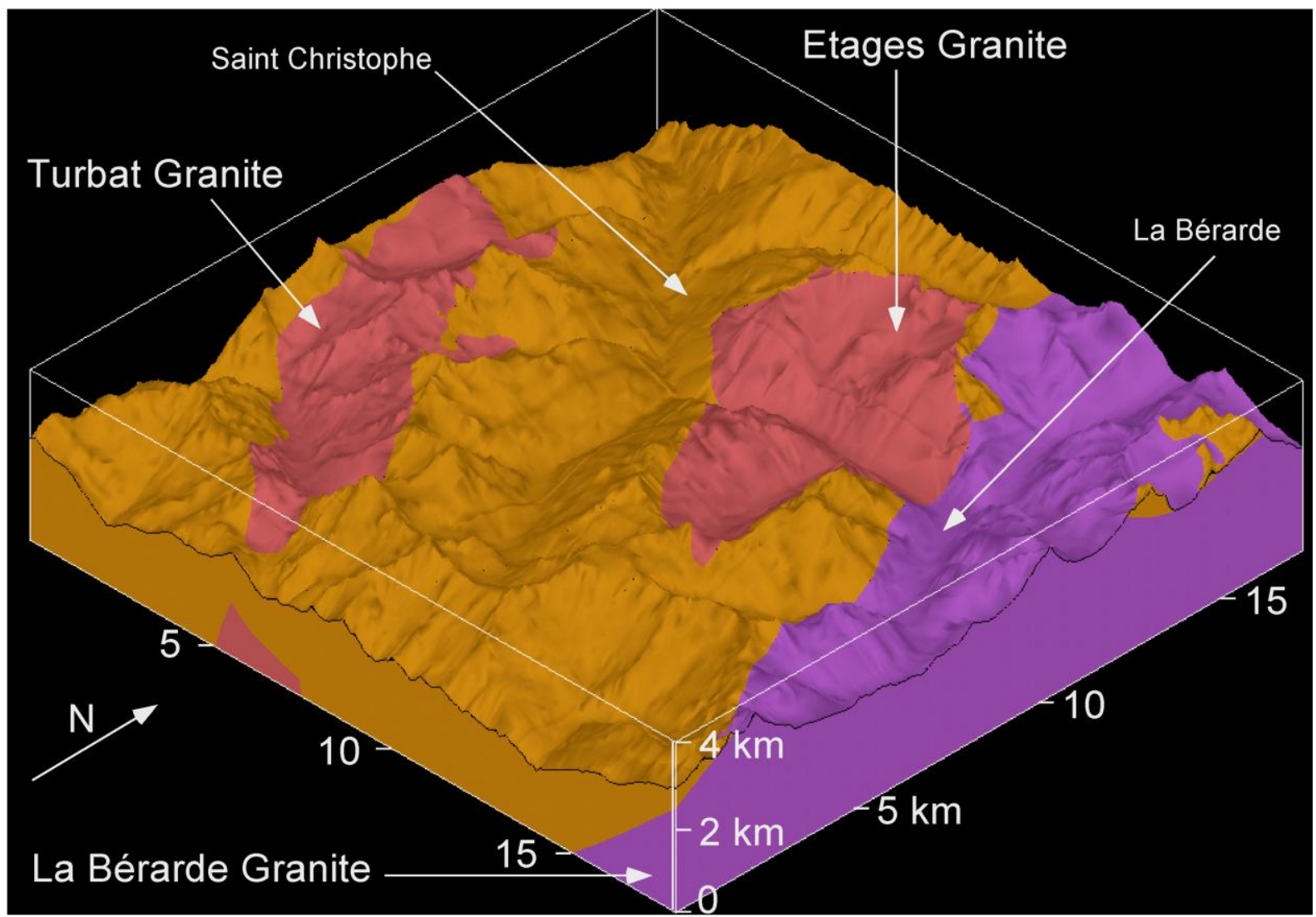

Figure V 


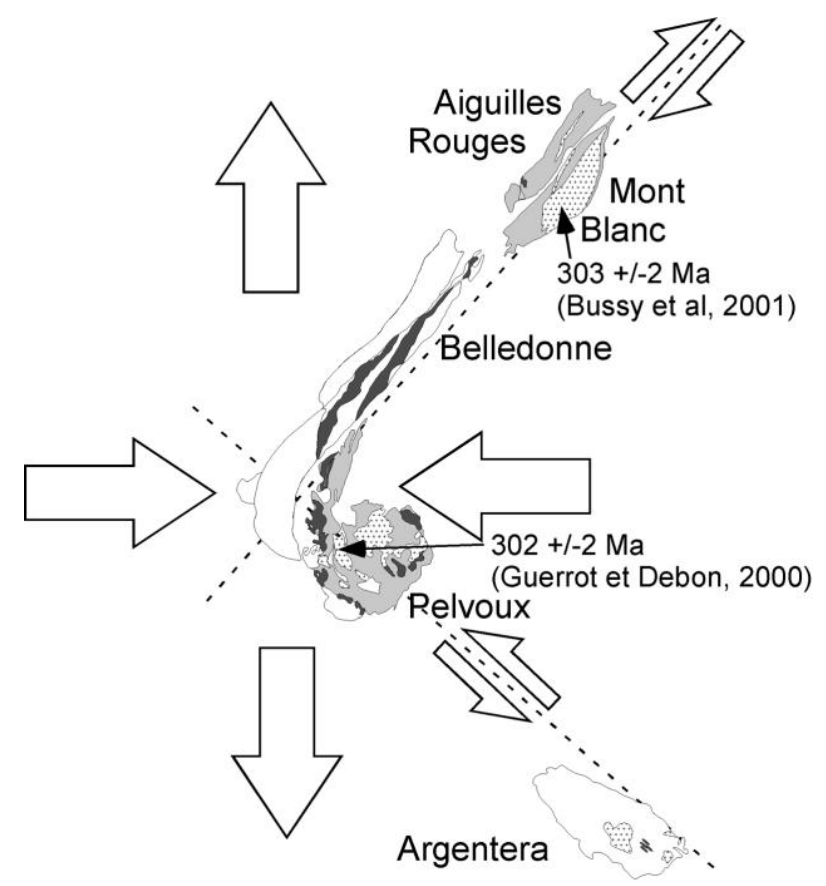

Figure VI 